\title{
Big brother is teaching you - Schule total digital?
}

\author{
Matthias Burchardt \\ Universität zu Köln, Institut für Bildungsphilosophie, Anthropologie und \\ Pädagogik der Lebensspanne, Allgemeine Pädagogik, Albertus-Magnus-Platz, \\ D-50931 Köln \\ m.burchardt@uni-koeln.de
}

\begin{abstract}
The article examines the political narratives and framework conditions of current discourses of digitization trends, criticizing them systematically by means of exemplary phenomena. The educational consequences of political programs are distinguished in eight ways. It is discussed in detail what consequences arise from the mere acquisition of equipment, what it would mean to replace teachers with algorithms, and finally, which power dispositions are established in the entanglement of economy and cybernetics by the concept of governance. A final consideration provides arguments for serenity in dealing with the hype of digitization, as essential content and skills conveyed at school can remain the same even in times of technological transformation, enabling students to deal responsibly with digitization.
\end{abstract}

»Alarm in den Schulen: Die Computer kommen. Computer in alle Schulen, alle Schulen an die Computer - dieses Programm wollen die Kultusminister zügig verwirklichen. Noch fehlt es an Rechnern und an Lehrern, die mit ihnen umgehen können. Auch gibt es Widerstand« (Spiegel 1984).

Wir schreiben das Jahr 1984. Das Orwell-Jahr. Der Spiegel titelt im Heft 47: »Revolution im Unterricht - Computer wird Pflicht«. Die maschinelle Datenspeicherung, -verarbeitung und -kommunikation dringt auf dem Wege der bezahlbaren Heimcomputer in die Schulen und Kinderzimmer vor. Der Spiegel prognostiziert den Einsatz von Robotern zur Tradition von Liedgut: »Es wird über kurz oder lang neben Computern auch >Hardware< anderer Art für die Schüler geben. Ein Vorläufer ist der aus den USA importierte Roboter >hero 1<, der Arbeit nur zu Unterrichtszwecken simuliert. Er bewegt sich ferngesteuert oder vorprogrammiert, greift mit seinem Arm Gegenstände, kann dank 
seiner Sensoren auch `sehen « und `hören<, singt das Volkslied `Old MacDonald had a farm< und erklärt sich in 50 Sätzen selbst. Er kostet brutto 10 ooo Mark und auch mit Rabatt wäre er für deutsche Schulen zu teuer, vermutlich erst in einigen Jahren werden die Roboter den Computern folgen« (ebd.).

Mit dem Abstand von über 30 Jahren wirken diese vollmundigen Prognosen anrührend naiv, so dass man geneigt ist, auch die aktuelle Digitalisierungsrhetorik als reine Medienhysterie abzutun: »Kinder reagieren besser, wenn sie von einem Roboter korrigiert werden, sagt Arvid Kappas [Psychologe, Jacobs-University] [...] Der Roboter wird nicht verbeamtet und geht nicht in Ruhestand. Er wird nicht ungeduldig, er ist nicht ungerecht, er kann keinen schlechten Tag haben. Der Schulroboter wird fortsetzen, was jetzt schon beginnt. [...] Ein Roboter wäre zunächst nur eine Hülle, in der die Programme laufen, er würde ihnen aber noch eine weitere Ebene hinzufügen, erklärt Kröger [Prof. am KIT in Karlsruhe]: Er könnte lächeln, wenn ein Kind etwas richtig macht. Man könnte ihn anfassen. Er könnte sich zu ihm bewegen« (ebd.).

Vor dem Hintergrund der jüngeren Geschichte erscheint die aktuelle Digitalisierungsdebatte wie ein Déjà-vu. Jörg Dräger von der Bertelsmann Stiftung ruft die »Digitale Bildungsrevolution « aus und die Bundesministerin für Bildung und Forschung Wanka startet eine »Bildungsoffensive für die digitale Wissensgesellschaft« (BMBF 2016a), lanciert mit den poetischen Worten: »Einmaleins und ABC nur noch mit dem PC!«. Beraten wird dieses Projekt unter anderem auf der Plattform »Digitalisierung in Bildung und Wissenschaft «. Der Zwischenbericht »Digitale Chancen nutzen. Die Zukunft gestalten.« (вм вғ 2016b) stellt fest, dass die Digitalisierung unsere Gesellschaft transformiert und fordert Innovationen im Bildungsbereich, die sich in >digitalen Kompetenzen und einer >Bildungscloud niederschlagen sollen. Ein Blick auf die Akteure der Plattform zeigt, dass dort keine Erziehungswissenschaftler_innen, sondern hauptsächlich Branchenvertreter der IT-Lobby versammelt sind: Bitkom, Deutsche Telekom, SAP, Microsoft, Scheer Group usf.

Das Narrativ des >Digitalen Wandels< bemüht dabei dieselbe Alternativlosigkeitsrhetorik wie die Globalisierung, so als wäre das demokratische Gemeinwesen einem Naturereignis ausgeliefert, vor dem man sich entweder schützen muss oder besser noch: aus dem man Profit schlagen kann. Die Hochglanzbroschüre der Ministerin wirft die Frage auf, ob dieser Wandel nicht just von den Akteuren betrieben wird, die ihre beratenden Dienste dem Gemeinwesen auf dieser Plattform so selbstlos zur Verfügung stellen. Da nicht gewährleistet ist, dass wir in 30 Jahren ebenso amüsiert auf die Allmachtsphantasien der Computerlobby schauen wie heute, wenn wir über den 
singenden Roboter aus dem Spiegel schmunzeln, sollen zur Vorbereitung und Orientierung des pädagogischen Widerstandes gegen eine Umsteuerung des Bildungssystems einige grundsätzliche Überlegungen zur Digitalisierung von Bildung angestellt werden. Dabei müssen im Sinne des Erkenntnisinteresses systematische und politische Überlegungen miteinander verschränkt werden. Dies geschieht aus der erfahrungsgesättigten Einsicht, dass pädagogische Theorien und Praxismodelle nicht allein dem >zwanglosen Zwang des besseren Arguments< erwachsen, sondern auch als Effekte von personaler und diskursiver Macht eingeschätzt werden müssen.

\section{Digitalisierung von Bildung}

Die Diskussion über die zu Beginn genannten Initiativen und Programme wird durch unscharfe Begriffsbezeichnungen erschwert. Wenn im Folgenden gegen die >Digitalisierung von Bildung < argumentiert wird, geht es ausdrücklich nicht um gute Arbeitsgeräte für Lehrer_innen, den Einsatz von Medien nach Maßgabe fachdidaktischer Erwägungen oder um medienpädagogische Unterrichtseinheiten, die emanzipatorische Ziele verfolgen.

Es lassen sich mindestens acht Deutungen aufweisen und unterscheiden, die theoretisch, ethisch, politisch und pädagogisch einer eigenen Beurteilung zugeführt werden müssten, Differenzierungen die in der gegenwärtigen Debatte oft untergehen:

1. Anschaffung von Geräten wie Smartboards, Tablets und Laptops

2. Bereitstellung von Infrastruktur und Software zur Vernetzung in und von Schulen: WLAN, Schulen ans Netz, Lernplattformen, Untis, Logineo, usf.

3. Digitale Codierung, Präsentation und Distribution der Lehr- und Lerninhalte statt analoger Medien

4. Medienpädagogische Nutzung von Digitalen Medien

5. Übertragung von traditionellen Lehrer_innenfunktionen auf Lernsoftware

6. Umerziehung von Schülern_Schülerinnen zum selbstgesteuerten Lernen

7. Ausrichtung der schulischen Bildungsziele auf Erfordernisse der digitalisierten Arbeitswelt (Industrie 4.0)

8. Kybernetische Steuerung der Schulorganisation durch Bildungscontrolling und Governance

Was in den Broschüren und Regierungsprogrammen kaum vorkommt, ist dagegen die Option einer Bildung am Gegenstand des Digitalen: das 
kybernetische Modell, Big Data, Netzpolitik, Technikfolgenabschätzung, Kryptographie, Telematik, Autoren, Redaktions- und Expertensysteme, autonome Tötungsentscheidungen von Drohnen, sozialkybernetische Steuerung der öffentlichen Meinung oder einzelner Personen beispielsweise wären sicher geeignete Themen, die in verschiedenen Fächern einen Anlass oder Horizont zur politischen oder persönlichen Bildung beisteuern könnten.

Im Rahmen dieses Artikels kann nicht jeder einzelne Punkt einer umfangreichen Untersuchung zugeführt werden, deshalb sollen vor allem vier übergreifende Aspekte diskutiert werden: Der vermeintlich neutrale Instrumentalcharakter der digitalen Medien, die trivial-kybernetische Umsteuerung des Lernens, die Ersetzung der Lehrperson durch Lernsoftware und schließlich die Machtkonstellation der Governance, die sich im Bildungscontrolling niederschlägt.

Wie kann es sein, dass es immer noch Klassen ohne Smartboards und Tablets gibt? Sollte der Anachronismus der >Kreidezeit` nicht endlich überwunden werden? Zur Illustration dieser Forderung wird bisweilen auf das >Schläferargument< zurückgegriffen. Dabei handelt es sich um eine Gedankenexperiment: Stellen Sie sich vor, man würde im Jahr 1817 einen Menschen in den Tiefschlaf versetzen und heute wieder aufwecken. Alles wäre ihm fremd, wenn er durch unsere Welt wanderte: Mobilität, Gesundheitswesen, Energiewirtschaft, Kommunikations- und Kriegsgeräte haben aufgrund des zivilisatorischen Fortschritts ein anderes Gesicht als vor 20o Jahren. Wenn der >Schläfer< allerdings eine Schulklasse besuchen würde, wäre ihm alles seltsam vertraut. Eine in der Sache und ihrer Vermittlung qualifizierte Lehrperson behandelt in einem dafür vorbereiteten Raum mit einer Gruppe von jungen Menschen Themen, um diese auf dem Weg der Bildung in die Welt einzuführen. Die Pointe dieser Erzählung besteht nun in der Frage: Wo ist denn hier der Fortschritt? Warum hängt da noch eine Tafel und kein Smartboard, warum steht da eine Lehrkraft und kein Roboter? Wieso schauen die Schüler_innen einander an und nicht auf den Bildschirm ihrer Tablets?

Vielleicht kann man die suggestive Kraft des Bildes am leichtesten brechen, wenn man es weiterspinnt: Was wäre, wenn der `Schläfer seinen Weg durch unsere Gegenwart fortsetzen und in einem Schlafzimmer landen würde, um dort Menschen bei ihrem Fortpflanzungsverhalten zu betrachten? Auch da hat sich im Wesentlichen nichts geändert, außer dass auf dem Nachttisch vielleicht einige Produkte der Erotik-Industrie liegen. Diese bringen vielleicht die 
Akteure ein wenig in Schwung, sind aber nicht die Ursache für die Entstehung von menschlichem Nachwuchs.

Der zugegebenermaßen drastische Vergleich kann zeigen, dass es anthropologische Grundlagen gibt, die - unabhängig vom technischen Stand einer Kultur - für das Gelingen von Fortpflanzung und Lernen unverzichtbar sind. Gegen eine Anreicherung der Grundsituation ist zunächst nichts einzuwenden, solange die hinzugezogenen Elemente nicht zur Perversion des eigentlichen Zwecks führen.

Die aktuelle bildungspolitische Situation scheint dagegen von einem digitalen Fetischismus geprägt zu sein, vom Glauben, dass Geräte und Software das Problem der Bildung von selbst und besser zu lösen in der Lage wären, als es die traditionelle Pädagogik konnte. >Wie kriegen wir die Geräte in die Schule?< lautet dann die Frage, so als sei die pädagogische Praxis von den Medien her zu denken und nicht der Medieneinsatz von der didaktischen Entscheidung her. Doch Tablets und Smartboards sind kein Selbstzweck, sie können hilfreich oder hinderlich sein. Beurteilen muss dies die Lehrperson.

Dabei müsste man schon aus der Erfahrung mit den Sprachlaboren und angesichts der immer kürzer werdenden Produktzyklen skeptisch werden: HighTech von heute ist zwangsläufig der Elektroschrott von morgen. In Anbetracht der knappen Personalsituation und der bedauernswerten Bausubstanz vieler Schulen böten sich dringlichere Investitionen an.

Hinzu kommt, dass der kindliche Alltag ohnehin durch digitale Endgeräte geprägt ist. In der BLIKK-Studie diagnostizieren Kinder- und Jugendärzte im Auftrag der Drogenbeauftragten der Bundesregierung den Rückgang des Lesens, die Hemmung der Sprachentwicklung, soziale Isolation, die reduzierte Fähigkeit zur Empathie, Suchtverhalten, Schlafstörung usf. (Die Drogenbeauftragte der Bundesregierung 2017).

Die Digitalisierungsbefürworter_innen im Bildungssektor bestreiten nun gar nicht die Gefahren oder Risiken, die von den neuen Technologien ausgehen. Sie machen sie sogar zum Argument für die Digitalisierungsoffensive. Digitale Medien, auch in ihrer schädlichen Wirkung, seien nun einmal Teil der gegebenen und zukünftigen Lebenswelt der Kinder und müssten deshalb auch in der Schule vorkommen. Dabei handelt es sich um einen naturalistischen Fehlschluss, wie an einem Gegenbeispiel leicht ersichtlich wird, wenn man etwa an Schulen in unsicheren Gegenden fordern würde, Kinder an Waffen auszubilden, weil diese nun einmal zu ihrer Lebenswelt gehörten. Aus dem Sein folgt nicht das Sollen, denn dazu bedarf es eines ethisch und pädagogisch wertenden Urteils, nach dem man hier wohl eher Friedenspädagogik auf den Lehrplan setzen würde als den Umgang mit scharfer Munition. 
Neben dem naturalistischen Fehlschluss unterläuft den Befürwortern ein weiterer Denkfehler: Sie führen den realen oder potentiellen Schaden auf einen missbräuchlichen Umgang mit der Technik zurück, die >an sich ‘ wertneutral sei (vgl. Heesen 2016, S. 27ff). Digitalisierung von Bildung, so wird dann gefolgert, sei notwendig, um diesen Missbrauch zu verhindern. Diese Einschätzung mag einer guten Absicht entspringen, ist aber theoretisch naiv und politisch uninformiert und deshalb gefährlich. In Gerätschaften realisiert sich die technische Rationalität, welche Ursache-Wirkungs-Prinzip in eine Mittel-Zweck-Relation übersetzt. Ganz im Sinne von Hegels Dialektik von >Herrschaft und Knechtschaft< (vgl. Hegel 1988, S. 127-136.) ist die Verfügungsgewalt des Nutzers über seine Werkzeuge nur im Rahmen bzw. in Form einer Unterwerfung gegeben.

Es sollte deutlich geworden sein, dass die Entstehungsbedingungen der technischen Instrumente jeweils schon von Macht und Interessen geprägt sind und diese Konstellationen auch den Geräten einschreiben. Die Wunschvorstellung vom Computer als einem neutralen Mittel gleicht einem Techno-Rousseauismus, weil man so tut, als könne das Gerät in einem QuasiNaturzustand unabhängig von Kultur, Geschichte, Wirtschaftsweise und Macht gedacht und gereinigt von allen impliziten Zweckmäßigkeiten (causa finalis, TELOS) genutzt werden. Die kontrafaktische Fiktion einer reinen Instrumentform ignoriert jedoch, dass es Geräte - ebenso wie den Menschen nur in Geschichte, Gesellschaft, Ökonomie und Macht gibt. Die Instrumentenform hat stets den Charakter einer Machtform, die uns in eins ermächtigt und übermächtigt.

\section{$3 \quad$ Computer als Lehrer}

Die Abkopplung von der Lehrperson im selbstregulierten Lernen der Neuen Lernkultur (Burchardt 2016) bereitet eine Ankopplung an das digitale Endgerät und den Algorithmus vor. Dräger und Müller-Eiselt preisen die Vorzüge der Software Knewton und lassen den Hersteller zu Wort kommen: »Knewton durchleuchtet jeden, der das Lernprogramm nutzt. Die Software beobachtet und speichert minutiös, was, wie und in welchem Tempo ein Schüler lernt. Jede Reaktion des Nutzers, jeder Mausklick und jeder Tastenanschlag, jede richtige und jede falsche Antwort, jeder Seitenaufruf und jeder Abbruch wird erfasst. `Jeden Tag sammeln wir tausende von Datenpunkten von jedem Schüler sagt Ferreira stolz. Diese Daten werden analysiert und zur Optimierung der persönlichen Lernwege genutzt. Komplexe Algorithmen schnüren individuelle 
Lernpakete für jeden einzelnen Schüler, deren Inhalt und Tempo sich fortlaufend anpassen, bei Bedarf im Minutentakt. [...] Schon heute berechnet Knewton zuverlässig die Wahrscheinlichkeit richtiger und falscher Antworten sowie die Note, die ein Schüler am Ende eines Kurses erreichen wird. Eines Tages braucht es wohl keine Prüfungen mehr; der Computer weiß bereits, welches Ergebnis herauskommen wird «(Dräger/Müller-Eiselt 2015, S. 24f).

Das Versprechen der Digitalisierung besteht in der Individualisierung von Lerndiagnostik, Stoff- und Aufgabenauswahl, die mittels Algorithmen effizienter und effektiver zu leisten seien, als von jedem Klassen- oder Kurslehrer. Ob diese versprochene oder sogar tatsächliche Funktionsoptimierung von Lernen auch eine bessere Bildung bedeutet, kann mit guten Gründen bezweifelt werden. Was heißt es, wenn Strukturmomente des Unterrichts, wie etwa die soziale Beziehung zwischen den Schülern_Schülerinnen und Lehrer_innen oder unter den Schülern_Schülerinnen ausgeklammert werden? Wenn die gemeinsame pädagogische Situation einer ko-intentionalen Sachvergegenwärtigung aufgehoben wird? Wenn Methodenwahl, Thematisierungshinsicht, Formatierung der Aufgaben, Motivation, Leistungserhebung und -beurteilung nicht mehr die Frage pädagogischer Urteilskraft oder persönlicher Verantwortung sind? Möglicherweise kann der Algorithmus >objektiver< bewerten als der_die Lehrer_in aus Fleisch und Blut. Allerdings sind die Maßstäbe und Standards der Software auch nur Ausdruck von Subjektivität, nämlich der Subjektivität der Programmierer_innen und Redakteure_Redakteurinnen. Und: Verfügt der Automat über das, was man früher mit Herbart den >pädagogischen Takt $<$ nannte? Kann er auch eine >pädagogische Note geben? In einem Unterrichtsgespräch war es für alle Beteiligten prinzipiell jederzeit möglich thematisch, methodisch oder auf der Beziehungsebene zu intervenieren. Aus einer Sachfrage >Was hat es mit der menschlichen DNA auf sich? $<$ muss situativ auch eine Wertfrage werden können: >Ist das Klonieren von Menschen legitim?< Bedeutet eine Ermächtigung der Software nicht eine stärkere Repression als sie jemals durch einen wilhelminischen Rohrstocklehrer möglich gewesen ist? Gegen den anonymen Algorithmus gibt es kein Aufbegehren und vor der Überwachung durch Big Data kein Verstecken.

Bisher sind die Befürworter der Digitalisierung durch Ersetzung des Lehrers_ der Lehrerin durch Software noch den Beweis einer Überlegenheit oder auch nur Gleichwertigkeit gegenüber gutem Unterricht schuldig geblieben. Es kann auch bezweifelt werden, ob dieser Nachweis zu erbringen ist. Sollte dies irgendwann gelingen, stellen sich immer noch die Fragen: Wollen wir so leben? Heiligt der Zweck die Mittel? 
Die Software >Knewton` vermittelt nicht nur Wissen, sondern erhebt auch Wissen über die Schüler_innen und ist insofern ein Instrument eines digitalen Panoptismus. Ohne jede pädagogische Theorie werden durch Big Data mittels statistischer Verfahren Cluster, Korrelationen, Normalverhalten, Abweichungen extrahiert, die dann dazu dienen, ein diagnostisches, manipulatives und prädiktives Herrschaftswissen bereitzustellen. Diese Daten können zur Optimierung von Lernen genutzt werden, wobei sicherlich die Frage zu diskutieren wäre, worin dieses Optimum denn bestehen solle. Die Frage nach dem Sinn ist jedenfalls eine andere als die nach der Funktion und kann entsprechend von der instrumentellen Vernunft nicht beantwortet werden.

Die Digitalisierungsoffensive der Ministerin ist dessen ungeachtet ein Projekt des Datensammelns und des >data miningく, des Auswertens eines möglichst unendlich großen Datenkorpus. Das politisch-kybernetische Projekt soll explizit die »strategische Organisationsentwicklung unterstützen« (вм вF 2016a, S. 25). Die Ziele der digitalen Transformation für das Jahr 2030 werden im Indikativ Präsens als Erreichte dargestellt, so als hätten die Akteure, über die hier verfügt wird, keine eigene Haltung oder Gestaltungshoheit in diesen Fragen: »Alle Leiterinnen und Leiter von Bildungseinrichtungen verfügen über die für die Umsetzung digitaler Bildung notwendigen organisatorischen, technischen und Management-Kompetenzen. Alle Lehrkräfte setzen das Lehren und Lernen mit digitalen Medien im Fachunterricht um. Die im Rahmen des Lernens mit digitalen Medien gewonnenen Erkenntnisse werden zur effizienteren Steuerung pädagogischer, personalwirtschaftlicher und bildungspolitischer Prozesse genutzt. Die im Rahmen des Lernens mit digitalen Medien gewonnenen Erkenntnisse zum Lernprozess haben neue Daten und Grundlagen für die empirische Bildungsforschung geliefert« (ebd.).

Die informationelle Vernetzung verknüpft individuelle und kollektive, pädagogische und organisationelle Aspekte unter einer kybernetisch-managarialen Perspektive: »Die digitale Bildung hält nicht nur für die einzelnen Bildungseinrichtungen, sondern zugleich für die Bildungsverwaltung als Ganzes neue Chancen bereit. So können die unter dem Vorzeichen der learning analytics gewonnenen Daten zugleich genutzt werden, um pädagogische, personalwirtschaftliche und bildungspolitische Prozesse effizienter zu steuern [...] (ebd.).

Im Hintergrund des sog. >Bildungscontrollings « steht die Herrschaftspraxis der >Governance $<$, die sich anschickt >Bildungspolitik $<$ in einem tatsächlich politischen Sinne zu verdrängen und durch ein kennziffern-gestütztes und 
sollwert-orientiertes technokratisches Steuern zu ersetzen: Das Streben nach guten Rankingplätzen, Akademiker_innenquoten, Drittmitteleinwerbungen, Publikationsratings im Umfeld eines wettbewerblichen Umfelds und managerialer Selbstführung, überführt die handelnden Akteure der Bildungspolitik und der Bildungseinrichtungen in ein Kraftfeld, in dem die Frage nach widerstreitenden Sinn- und Wertbestimmungen hinfällig geworden ist. Die vermeintliche Objektivität der Zahlen als Überwindung von Ideologisierungen zu feiern wäre allerdings sehr kurzsichtig, weil das Zahlenregime und das prozedurale Betriebssystem der Optimierungstechniken seinerseits kryptonormativ determiniert sind.

»Mit einem Wort, die Governance verbreitet eine entpolitisierende Erkenntnistheorie, Ontologie und eine Gesamtheit von Praktiken. Da ihre Ausrichtung weich, inklusiv und technisch ist, verscharrt die Governance strittige Normen und strukturelle Schichtenbildungen (wie zum Beispiel Klassen) sowie die Normen und Ausschlüsse, die durch ihre Verfahren und Entscheidungen in Umlauf gesetzt werden. Sie integriert Subjekte in die Zwecke und Bahnen der Nationen, Betriebe, Universitäten oder anderer Gebilde, die sich ihrer bedienen. Im öffentlichen Leben verdrängt die Governance liberal-demokratische Anliegen mit Bezug auf Gerechtigkeit durch technische Problemformulierungen, Fragen nach dem, was recht ist, durch Fragen nach der Effizienz, selbst Fragen nach dem, was legal ist, durch solche nach der Effektivität« (Brown 2015, S. 17).

Die Digitalisierung von Bildung fungiert letztlich als Element der postpolitischen Schulsteuerung und Organisationsentwicklung, sie errichtet über Schüler_innnen, Lehrerkollegien und Schulverwaltungen ein Regime der Kontrolle im Sinne von Sichtbarkeit und Steuerung. Freiheits- und Gestaltungsspielräume von Lehrpersonen, Wissenschaftlern_Wissenschaftlerinnen oder sogar Politikern_Politikerinnen sind funktionalistisch präokkupiert und damit de facto abgeschafft.

Die beste Antwort auf die digitalen Zeiten: Rückbesinnung auf traditionelle Bildung.

Muss durch den Digitalisierungstrend wirklich alles anders werden? Eine wesentliche Hinsicht, an der sich die Beurteilung von Inhalten und Zielen der schulischen Bildung orientiert, kann die Zeitperspektive bilden.

Dabei kommt es darauf an, die kurzfristig erforderlichen Qualifikationen und Kenntnisse (I.) von den längerfristig bedeutsamen kognitiven und praktischen Konzepten (II.) zu unterscheiden und diese wiederum von den übergreifenden Zielen der allgemeinen Menschenbildung (III.). 


\begin{tabular}{|c|c|c|c|}
\hline Dimensionen & Beispiele & zeitl. Relevanz & Erwerb \\
\hline $\begin{array}{l}\text { I. Pragmatische } \\
\text { Kompetenzen } \\
\text { und funktionales }\end{array}$ & $\begin{array}{l}\text { Textverarbei- } \\
\text { tung, Präsenta- } \\
\text { tionssoftware }\end{array}$ & $\begin{array}{l}\text { kurzfristig in } \\
\text { Abhängigkeit zum } \\
\text { zivilisatorischen }\end{array}$ & $\begin{array}{l}\text { leicht und schnell } \\
\text { vermittelbar auf } \\
\text { der Grundlage }\end{array}$ \\
\hline Wissen & $\begin{array}{l}\text { Suchmaschinen, } \\
\text { Programmierung }\end{array}$ & Fortschritt & von II. \\
\hline $\begin{array}{l}\text { II. Kognitive } \\
\text { und praktische } \\
\text { Konzepte }\end{array}$ & $\begin{array}{l}\text { Lesen, Schreiben, } \\
\text { Rechnen, Beurtei- } \\
\text { len, Imaginieren }\end{array}$ & $\begin{array}{l}\text { langfristig in } \\
\text { Abhängigkeit } \\
\text { von kulturellen }\end{array}$ & $\begin{array}{l}\text { Übung und } \\
\text { Unterricht über } \\
\text { viele Jahre im }\end{array}$ \\
\hline $\begin{array}{l}\text { III. Allgemeine } \\
\text { Menschenbildung }\end{array}$ & $\begin{array}{l}\text { Sinnstiftung, Weis- } \\
\text { heit, Werte, Verant- } \\
\text { wortung, Glück }\end{array}$ & $\begin{array}{l}\text { Paradigmen } \\
\text { grundlegend in Ab- } \\
\text { hängigkeit von der } \\
\text { conditio humana }\end{array}$ & $\begin{array}{l}\text { Horizont von III. } \\
\text { Lebensaufgabe }\end{array}$ \\
\hline
\end{tabular}

Es ist schlicht falsch, dass die Wissensgesellschaft oder die Digitalisierung tradierte Bildung überflüssig machen würden. Lebensbewältigung und Persönlichkeitsbildung beziehen sich im Grunde seit der Antike auf dieselben Quellen von Grundfähigkeiten und -fertigkeiten, die historischen Unterschiede sind eher oberflächlich: $\mathrm{Ob}$ ich nun einen theologischen Text in einem Kodex oder eine Anleitung zum Change-Management auf dem E-Reader lese, in beiden Fällen brauche ich die Fähigkeiten des Lesens, Verstehens und Beurteilens, und bin mit der Frage konfrontiert: Was bedeutet das für mich und die Gemeinschaft? D. h. die Ebenen II. und III. bleiben gleich, während natürlich ganz andere Mikrotechniken in der Mediennutzung auf der Ebene I. erforderlich werden. Diese oberflächlichen Kulturtechniken wandeln sich zwar schneller im Takt des zivilisatorischen Fortschritts, sind aber auch wesentlich leichter zu erlernen als die grundlegenden: Ein Schulkind benötigt viele Jahre, um Lesen und Schreiben zu lernen (II.) und damit die sprachliche Welt in Verstehen und Ausdruck verantwortlich bewohnen zu können (III.). Der mittelalterliche Mönch dagegen hätte wohl kaum mehr als zehn Minuten gebraucht, um zu begreifen, wie man einen E-Reader nutzt.

Es dürfte deutlich geworden sein, dass die Schule in den Bereichen II. und III. gelassen bleiben kann, weil eigentlich nur im Bereich I. Veränderungen erforderlich sind. Hier aber kann es auch nur um Exemplarisches und Grundlegendes gehen, eine Abbildung des gesamten Spektrums technischer Anwendungen ist selbst in der beruflichen Bildung weder möglich noch sinnvoll. 


\section{Literatur}

Burchardt, Matthias (2016): Selbstgesteuertes Lernen - Roboter im Klassenzimmer. In: Klaus Zierer/Joachim Kahlert/Matthias Burchardt (Hrsg.): Die pädagogische Mitte. Bad Heilbrunn: Klinkhardt, S. 121-134.

Brown, Wendy (2015): Die schleichende Revolution. Wie der Neoliberalismus die Demokratie zerstört. Frankfurt/Main: Suhrkamp.

Dräger, Jörg/Müller-Eiselt, Ralph (2015): Die digitale Bildungsrevolution. München: DVA.

Heesen, Jessica (Hrsg.) (2016): Handbuch Medien- und Informationsethik. Stuttgart: Metzler.

Hegel, Georg Wilhelm Friedrich (1988): Phänomenologie des Geistes. Hamburg: Meiner.

Schirrmacher, Frank (2009): Payback (3. Aufl.). München: Blessing.

Wiener, Norbert (1964): Mensch und Menschmaschine [1952]. Frankfurt: Athenäum.

\section{Internetquellen}

Barlow, John P. (1996): Unabhängigkeitserklärung des Cyberspace. URL: https://www .heise.de/tp/features/Unabhaengigkeitserklaerung-des-Cyberspace-3410887.html (Datum des letzten Abrufs: 15. Januar 2018).

вм в (2016а): Bildungsoffensive für die digitaleWissensgesellschaft. Oktober 2016. URL: https://www.bmbf.de/files/Bildungsoffensive_fuer_die_digitale_Wissensgesells chaft.pdf (Datum des letzten Abrufs: 15. Januar 2018).

вмвғ (2016b): Digitale Chancen nutzen. Die Zukunft gestalten. November 2016. URL: https://www.bildung-forschung.digital/files/BMBF_Digitale_Bildung_Zwischenbe richt_A4_webRZ.pdf (Datum des letzten Abrufs: 15. Januar 2018).

Die Drogenbeauftragte der Bundesregierung (2017): Ergebnisse der BLIKK-Studie 2017 vorgestellt. Presseerklärung vom 29.5.2017. URL: http://www.drogenbeauftragte .de/presse/pressekontakt-und-mitteilungen/2017/2017-2-quartal/ergebnisse -der-blikk-studie-2017-vorgestellt.html (Datum des letzten Abrufs: 15. Januar 2018).

Lübke,Friederike(2017):Wirsehenunsin derSchule.DieWelt.9.9.2017.URL:https:/www .welt.de/print/welt_kompakt/webwelt/article168734451/Wir-sehen-uns-in-der -Schule.html. (Datum des letzten Abrufs: 8. Januar 2017).

Spiegel (1984): Alarm in den Schulen: Die Computer kommen. Heft 47, 1984. URL: http://www.spiegel.de/spiegel/print/d-13512161.html (Datum des letzten Abrufs: 15 . Januar 2018). 